Curr. Drug Targ.. 2006 (7):893-909.

\title{
The Functions and Structure of ABC Transporters: Implications for the Design of New Inhibitors of Pgp and MRP1 to Control Multidrug Resistance (MDR)
}

Teodori E., Dei, S., Martelli, C., Scapecchi, S., Gualtieri, F. Abstract

Multidrug resistance (MDR) is a kind of acquired resistance of microorganisms and cancer cells to chemotherapic drugs that are characterized by different chemical structure and different mechanism of action. Classic MDR is the consequence of the over-expression of a variety of proteins that extrude the chemotherapic from the cell, lowering its concentration below the effective one. The ABC (ATP Binding Cassette) is a ubiquitous and important family of such transporter proteins. Members of this super family are present in mammals as well as in prokaryotic organisms and use ATP as the energy source to activate the extrusion process. P-glycoprotein (Pgp) and Multidrug Resistance Proteins (MRP1 and sister proteins) are the most important and widely studied members of ABC super family.

Our knowledge about the structures and functions of transporter proteins has definitely improved in recent years, following the resolution of the structure of bacterial pumps which opened the way to the building of homology models for the more complex Pgp and MRP. It can be anticipated that these results will have a strong impact on the design of more potent and safer MDR reverters.

A huge number of small molecules, many of natural origin, are able to reverse multidrug resistance by inhibiting the functions of Pgp, MRP1 and sister proteins and their action has been considered a possible way to reverse MDR. However, while a few compounds have reached clinical trials, none of them has, so far, been cleared for therapeutic use. Two main reasons are at the base of this difficulty: i) MDR is a complex phenomenon that may arise from several different biochemical mechanisms, with the consequence that inhibition of transporter proteins may be insufficient to reverse it; ii) the physiological role of Pgp and sister proteins requires more potent modulators with proper selectivity and pharmacokinetic in order to avoid unwanted side effects.

This paper first reviews the most recent discoveries on the structures and functions of the $A B C$ super family, in particular Pgp and MRP. Then, the medicinal chemistry of MDR reverters, in light of these findings, is discussed and the molecules that are presently in development are reviewed. 